\title{
Diabetes mellitus en contexto de enfermedad mitocondrial fenotipo Kearns-Sayre
}

\begin{abstract}
Carmen Yllera-Gutiérrez, Carlota Argüello-Martín, Lucía Meijide-Rodríguez, María Folgueras-Gómez, Silvia Suárez-Díaz UGC Medicina Interna. Hospital Universitario Central de Asturias. Oviedo (Principado de Asturias). España
\end{abstract}

Recibido: 11/11/2018

Aceptado: 01/01/2019

En línea: 31/01/2019

Citar como: Yllera-Gutiérrez C, Argüello-Martín C, Meijide-Rodríguez L, Folgueras-Gómez M, Suárez-Díaz S. Diabetes mellitus en contexto de enfermedad mitocondrial fenotipo Kearns-Sayre. Rev Esp Casos Clin Med Intern (RECCMI). 2019 (Ene); 4(Supl 1): 3-4. doi: 10.32818/reccmi.a4s1a2.

Autor para correspondencia: Carmen Yllera-Gutiérrez.c.yllera.gutierrez@gmail.com

\section{Palabras clave \\ $\triangleright$ Diabetes mellitus \\ $\triangleright$ Enfermedad mitocondrial \\ $\triangleright$ Síndrome de Kearns-Sayre}

\begin{abstract}
Resumen
Paciente de 36 años con antecedente de diabetes mellitus, sordera neurosensorial y retinosis pigmentaria que tras consulta por ptosis palpebral de larga evolución y debilidad muscular progresiva es diagnosticada de miopatía mitocondrial fenotipo Kearns-Sayre. En dicho síndrome, es muy frecuente la aparición de endocrinopatías basadas en el déficit de energía para la secreción hormonal. La paciente fallece tras múltiples episodios de insuficiencia cardíaca por afectación miocárdica, siendo más frecuente en este síndrome la afectación de las fibras de conducción.
\end{abstract}

\section{Puntos destacados}

$\triangleright$ Presentamos un caso de una mujer con diagnóstico tardío de síndrome de Kearns-Sayre con antecedente de diabetes mellitus con necesidad de insulinización precoz.

$\triangleright$ Ésta podría haber sido la primera manifestación de su enfermedad mitocondrial.

\section{Introducción}

Las enfermedades mitocondriales son entidades poco prevalentes y con inicio a edades pediátricas cuya mayor afectación tiene lugar a nivel del tejido muscular, con la implicación cardíaca como mayor condicionante del pronóstico en estos casos?

El síndrome de Kearns-Sayre (SKS) se caracteriza por oftalmoplejia externa con ptosis, sordera neurosensorial y retinosis pigmentaria².

En función de las mutaciones descritas en cada entidad, pueden verse comprometidos distintos órganos, siendo la diabetes la endocrinopatía más frecuente, presente en el SKS hasta en el 10\% de los casos $^{3}$.

\section{Historia clínica: antecedentes, enfermedad actual y exploración física}

Paciente diagnosticada de diabetes mellitus (DM) a los 36 años (1990) en tratamiento con insulina premezclada desde 1997 para un total de 46 UI/día (0,88 UI/kg/día). Presenta también cofosis bilateral y degeneración retiniana desde la juventud. Sufrió cuatro abortos. Es remitida a la consulta (2007) por ptosis bilateral, objetivándose también hipotonía generalizada y reflejos osteotendinosos de miembros superiores abolidos. En resonancia magnética nuclear se evidencia atrofia olivopontocerebelosa, por lo que se completa el estudio con electromiograma en el que se observan alteraciones compatibles con miopatía, por lo que la paciente es diagnosticada de enfermedad mitocondrial con fenotipo de SKS.

\section{Pruebas complementarias}

Se efectúan las siguientes pruebas complementarias:

- Resonancia cerebral. Cuarto ventrículo muy aumentado de tamaño, aumento del espacio subaracnoideo a nivel de folias y vérmix cerebeloso. 
Aumento del espacio subaracnoideo en fosa posterior. Ventriculomegalia simétrica.

- Fondo de ojo (ambos ojos). Gran atrofia de epitelio pigmentario de la retina y coriocapilar, bien delimitado en polo posterior, sin anomalías vasculares visibles.

- Biopsia muscular:

- NADH con patrón interno de las fibras normal.

- ATPasa con patrón en mosaico característico.

- Citocromo-oxidasa con pérdida completa en fibras aisladas.

- Succinodeshidrogenasa con confirmación de hiperplasia mitocondrial focal.

No hay signos definitivos de mitocondriopatía pero sí signos indirectos, como pérdida de expresión de citocromo y aumento de mitocondrias subsarcomales. Se envía material al Hospital Universitario 12 de Octubre, en el que se detecta presencia de una mutación en heteroplasmia en el ADN mitocondrial, lo que confirma la sospecha diagnóstica de miopatía mitocondrial.

- Electromiograma. Potenciales de unidad motora voluntarios de duración dentro de límites normales, con incidencia de polifasia incrementada; patrones de esfuerzo mixto para activación submáxima en todos los músculos estudiados. La exploración neurofisiológica en la actualidad sugiere hallazgos compatibles con patrón miopático.

- Hemoglobina glicosilada:

- Enero de 2008: 8,4\%.

- $\quad$ Mayo de 2009: 8,7\%.

\section{Evolución}

Desde el diagnóstico, en 2007, la paciente precisa tres ingresos por infecciones respiratorias con descompensación de insuficiencia cardíaca, por lo que se realiza estudio de cardiopatía. En 2008, el ecocardiograma transtorácico es sugestivo de enfermedad de depósito con disfunción leve de ventrículo izquierdo; y, el siguiente año, se evidencia disfunción leve-moderada del ventrículo izquierdo.

En 2009, la paciente ingresa por cuadro de shock hipovolémico en contexto de gastroenteritis aguda y, tras reposición hídrica, entra en edema agudo de pulmón, con shock cardiogénico, falleciendo a la edad de 55 años.

\section{Diagnóstico}

DM en contexto de enfermedad mitocondrial.

\section{Discusión}

Si bien la clínica típica diagnóstica del SKS consiste en la oftalmoplejia externa con ptosis y la retinopatía pigmentaria, y se presenta en las primeras dos décadas de la vida, las endocrinopatías son también frecuentes y la DM se encuentra presente al diagnóstico de hasta el 10\% de los casos $^{2}$.

La afectación que confiere el pronóstico limitado en estos pacientes suele estar en relación con la afectación cardíaca que, en esta paciente, no es la característicamente descrita por afectación de las fibras de conducción, sino puramente miocárdica, con múltiples descompensaciones de insuficiencia cardíaca’.

La afectación diabética en los pacientes con enfermedad mitocondrial se basa en la necesidad del ATP para la secreción de insulina en los islotes pancreáticos, de forma que se produce un déficit en la secreción de insulina, no así de su producción y almacenamiento, siendo infrecuente la presencia de autoanticuerpos pancreáticos ${ }^{1-4}$. De esta forma, aunque la presentación de la diabetes hace necesaria la insulinización, otros fármacos no insulínicos, especialmente sulfonilureas, pueden tener cabida en el tratamiento de la diabetes relacionada con las enfermedades mitocondriales².

La DM en relación con enfermedades mitocondriales debe ser sospechada en diabetes de debut en la edad adulta, mal control glucémico desde el inicio del tratamiento y con sintomatología sistémica congruente con las enfermedades mitocondriales descritas ${ }^{2-4}$.

\section{Bibliografía}

1. Ho J, Pacaud D, Rakic M, Khan A. Diabetes in pediatric patients with Kearns-Sayre syndrome: clinical presentation of 2 cases and review of pathophysiology. Can J Diabetes. 2014 Aug; 38(4): 225-228. doi: 10.1016/j. jcjd.2014.04.003.

2. Chow J, Rahman J, Achermann JC, Dattani MT, Rahman S. Mitochondrial disease and endocrine dysfunction. Nat Rev Endocrinol. 2017 Feb; 13(2): 92-104. doi: 10.1038/nrendo.2016.151.

3. Laloi-Michelin M, Virally M, Jardel C, Meas T, Ingster-Moati I, Lombès A, Massin P, Chabriat H, Tielmans A, Mikol J, Guillauseau PJ. Kearns Sayre syndrome: an unusual form of mitochondrial diabetes. Diabetes Metab. 2006 Apr; 32(2): 182-186. doi: 10.1016/S1262-3636(07)70267-7.

4. Al-Gadi IS, Haas RH, Falk MJ, Goldstein A, McCormack SE. Endocrine disorders in primary mitochondrial disease. J Endocr Soc. 2018 Feb 19; 2(4): 361 373. doi:10.1210/js.2017-00434. 